\title{
The trace amine-associated receptor 1 modulates methamphetamine's neurochemical and behavioral effects
}

\section{Rachel Cotter ${ }^{1+}$, Yue Pei ${ }^{1,2+}$, Liudmila Mus ${ }^{3}$, Anja Harmeier ${ }^{4}$, Raul R. Gainetdinov ${ }^{3,5,6}$, Marius C. Hoener ${ }^{4}$ and Juan J. Canales ${ }^{2 *}$}

${ }^{1}$ Department of Psychology, University of Canterbury, Christchurch, New Zealand

2 Behavioural Neuroscience, School of Psychology, University of Leicester, Leicester, UK

${ }^{3}$ Department of Neuroscience and Brain Technologies, Istituto Italiano di Tecnologia, Genoa, Italy

${ }^{4}$ Neuroscience Research, Pharmaceuticals Division, F. Hoffmann-La Roche Ltd., Basel, Switzerland

${ }^{5}$ Skolkovo Institute of Science and Technology, Skolkovo, Moscow, Russia

${ }^{6}$ Faculty of Biology, St. Petersburg State University, St. Petersburg, Russia

\section{Edited by:}

Marianthi Papakosta, Pfizer, USA

Reviewed by:

Nick Andrews, Harvard Medical

School, USA

Cathy Fernandes, King's College

London, UK

\section{*Correspondence:}

Juan J. Canales, School of

Psychology, University of Leicester

Lancaster Road, LE1 9HN Leicester,

UK

e-mail: jjc30@leicester.ac.uk

tThese authors have contributed equally to this work.
The newly discovered trace amine-associated receptor 1 (TAAR1) has the ability to regulate both dopamine function and psychostimulant action. Here, we tested in rats the ability of R05203648, a selective TAAR1 partial agonist, to modulate the physiological and behavioral effects of methamphetamine (METH). In experiment 1, RO5203468 dose- and time-dependently altered METH-induced locomotor activity, manifested as an early attenuation followed by a late potentiation of METH's stimulating effects. In experiment 2, rats received a 14-day treatment regimen during which R05203648 was co-administered with METH. R05203648 dose-dependently attenuated METH-stimulated hyperactivity, with the effects becoming more apparent as the treatments progressed. After chronic exposure and 3-day withdrawal, rats were tested for locomotor sensitization. R05203648 administration during the sensitizing phase prevented the development of METH sensitization. However, RO5203648, at the high dose, cross-sensitized with METH. In experiment 3, RO5203648 dose-dependently blocked METH self-administration without affecting operant responding maintained by sucrose, and exhibited lack of reinforcing efficacy when tested as a METH's substitute. Neurochemical data showed that RO5203648 did not affect METH-mediated DA efflux and uptake inhibition in striatal synaptosomes. In vivo, however, RO5203648 was able to transiently inhibit METH-induced accumulation of extracellular DA levels in the nucleus accumbens. Taken together, these data highlight the significant potential of TAAR1 to modulate METH's neurochemical and behavioral effects.

Keywords: trace amine-associated receptor, methamphetamine, sensitization, self-administration, synaptosomes, microdialysis

\section{INTRODUCTION}

Methamphetamine (METH) is a widely abused and highly addictive psychostimulant drug. In the brain, METH elevates extracellular dopamine (DA) levels, an effect that is thought to underlie its potent motor and psychoactive actions (Fleckenstein et al., 2007). METH competes with DA for reuptake and causes reverse transport through the DA transporter (DAT) (Elliott and Beveridge, 2005; Goodwin et al., 2009). In addition, methamphetamine depletes monoamines from vesicular storage through the vesicular monoamine transporter-2 (VMAT2), resulting in increased DA in the cytoplasm available for reverse transport by the DAT (Sulzer et al., 2005; Fleckenstein et al., 2009). Apart from its direct interaction with the DA system, METH is also a potent agonist at the trace amine-associated receptor 1 (TAAR1) (Bunzow et al., 2001; Reese et al., 2007). TAAR1 belongs to a family of G-protein coupled receptors that is activated by trace amines (TAs), a group of endogenous amines often referred to as "endogenous amphetamines" due to their ability to increase monoamine release via displacing monoamines from synaptic vesicles and reversing monoamine transporters on the plasma membrane (Berry, 2004). TAs have been implicated in brain reward and in the reinforcing properties of psychostimulants (Gilbert and Cooper, 1983; Shannon and Thompson, 1984), albeit their independent role as neurotransmitters was not recognized until TAAR1 was discovered and characterized (Borowsky et al., 2001; Bunzow et al., 2001). TAAR1 shares overlapping distribution in the brain with mesolimbic DA pathways (Borowsky et al., 2001; Lindemann et al., 2008), is co-localized with the DAT in a subset of DA neurons (Xie and Miller, 2007), and interacts with both the DAT and the D2 DA autoreceptor (D2R) to modulate DA transmission (Xie et al., 2007, 2008; Espinoza et al., 2011). These observations suggest that TAAR1 regulates DA activity and has the potential to serve as a pharmacological target to modulate DA dysregulation that results from chronic stimulant exposure.

Early evidence supporting the potential of TAAR1 to control DA function comes from studies on Taar1 knock-out mice 
which showed an elevated spontaneous firing rate of DA neurons in the ventral tegmental area (Lindemann et al., 2008) and increased DA release in the NAc (Leo et al., 2014), suggesting that TAAR1 is constitutively active or tonically activated by ambient levels of amines, including TAs, to negatively regulate DA activity. Those mutant mice also displayed enhanced sensitivity to psychostimulant-induced locomotor activity, conditioned place preference (Achat-Mendes et al., 2012) and striatal DA release (Wolinsky et al., 2007; Lindemann et al., 2008), supporting the role of TAAR1 in psychostimulant action. The recent development of several selective TAAR1 agonists has enabled a more direct assessment of TAAR1 functionality. While the TAAR1 full agonists, RO5166017 (Revel et al., 2011) and RO5256390 (Revel et al., 2012a), were able to inhibit the firing rate of dopaminergic neurons in the ventral tegmental area, the TAAR1 partial agonists, RO5203648 (Revel et al., 2012b) and RO5263397 (Revel et al., 2012a), and the antagonist, EPPTB (Bradaia et al., 2009), increased their firing frequency, demonstrating a strong modulation of DA activity by TAAR1 activation.

More recent studies have focused on examining the therapeutic-like effects of TAAR1 agonists on cocaine addiction. It was found that TAAR1 activation suppressed cocaine-induced locomotor hyperactivity (Revel et al., 2012b), behavioral sensitization and conditioned place preference (Thorn et al., 2014), and reduced cocaine self-administration (Revel et al., 2012b; Thorn et al., 2014), suggesting a blockade of both acute psychostimulating and long-term psychomotor sensitization effect of cocaine as well as cocaine's reinforcing property through pharmacological activation of TAAR1. Moreover, the two partial agonists, RO5203648 (Pei et al., 2014) and RO5263397 (Thorn et al., 2014) were able to block cocaine relapse, further supporting the development of TAAR1-based pharmacotherapies in cocaine addiction. Data on potential interactions between METH and TAAR1 is much more limited. METH and cocaine have different actions on DAT with the former producing strong DA releasing effects. More importantly, while TAAR1 has no affinity for cocaine, activation of TAAR1 by METH results in inhibition of DA uptake, enhancement of DA efflux and DAT internalization (Xie and Miller, 2009). The present work was aimed at further characterizing the effects of TAAR1 activation on key METH-related behavioral and neurochemical changes. We tested the ability of the partial agonist, RO5203648, to modulate METH-induced locomotor activity and behavioral sensitization, METH self-administration and substitution, and METH-stimulated striatal DA efflux in vitro and in vivo.

\section{MATERIALS AND METHODS SUBJECTS}

Male Long Evans rats were sourced from the University of Canterbury, the University of Otago and the Italian Institute of Technology and were 8-10 weeks-old when experiments began. Rats were generated from outbred stocks, with rats from different institutions or suppliers not bearing any direct relationship. For the behavioral experiments, all animals were housed in a temperature and humidity controlled colony room with a 12h light/dark cycle (lights off at 8 a.m.). Water and standard laboratory rat chow was given ad libitum at all times in all experiments except in the self-administration (S-A) experiment in which rats were given a maintenance diet (i.e., kept at $100 \%$ of their weight 7 days post-surgery) (Velazquez-Sanchez et al., 2011, 2013). To perform the $\left[{ }^{3} \mathrm{H}\right]$ dopamine uptake and efflux assays synaptosomes were prepared from brains of Wistar rats (F. Hoffmann-La Roche Ltd., Basel, Switzerland). Animal care and experimental protocols were conducted in compliance with the New Zealand Animal Welfare Act 1999, the Italian Ministry of Health (DL 116/92; DL 111/94-B) and European Community (86/609/EEC) directives, and the Swiss Federal and Basel Cantonal laws on animal research. All experiments were approved by the ethics committee affiliated to each institution. Power analysis was conducted as part of the applications for ethics approval to estimate the number of animals required per experiment, with $\alpha$ set at 0.05 and power at $90 \%$. Data from all animals was included in the statistical analyses.

\section{PHARMACOLOGICAL AGENTS}

Methamphetamine hydrochloride was obtained from BDG Synthesis (Wellington, New Zealand) and dissolved in $0.9 \%$ physiological saline for intraperitoneal (i.p.) injection and intravenous S-A. RO5203648 (partial TAAR1 agonist) was synthesized at F. Hoffman-La Roche Ltd. (Switzerland) and dissolved in $10 \%$ dimethylsulfoxide and $0.9 \%$ physiological saline.

\section{CATHETER IMPLANTATION SURGERY}

Rats used for the S-A experiment were anesthetized with Avertin (2,2,2-tribromoethanol, $12.5 \mathrm{mg} / \mathrm{ml}$, in $2.5 \%$ tertiary amyl alcohol, $2 \mathrm{ml} / 100 \mathrm{~g}$ of body weight, i.p.). The analgesic carprofen was administered before surgery $(5 \mathrm{mg} / \mathrm{kg}$, i.p.). Catheters (O/D $0.63 \mathrm{~mm}, \mathrm{I} / \mathrm{D} 0.30 \mathrm{~mm}$, Camcaths Cambridge, UK) were implanted into the right jugular vein, exiting dorsally between the scapulae. Analgesic and antiseptic cream was applied to the back and neck incision areas following suturing. To prevent infection rats were treated post surgically with daily injections of antibiotic (Cephalexin, $10 \mathrm{mg} / \mathrm{kg}$, s.c.) for 7 days. Catheters were flushed with heparinised saline $(0.1 \mathrm{ml}, 70 \mathrm{IU} / \mathrm{ml})$ before and after each S-A session.

\section{BEHAVIORAL PROCEDURES Locomotor activity}

Locomotor activity experiments were conducted in a set of four open field boxes made of black Perspex $(50 \times 40 \times 35 \mathrm{~cm})$. Locomotor activity was monitored and measured with a video tracking system and image analysis software (Viewpoint 2.5, Champagne au Mont D'Or, France) that provided automatic measures of traveled distance, trajectory and velocity of the subjects.

Six groups of rats ( $n=5-6$ per group) were habituated in the open field for $10 \mathrm{~min}$ for two consecutive days. During the test, rats were given a pretreatment of RO5203648 $(0,5,10 \mathrm{mg} / \mathrm{kg}$, i.p.) followed $15 \mathrm{~min}$ after by $\mathrm{METH}(0,0.75 \mathrm{mg} / \mathrm{kg}$, i.p.). $10 \mathrm{~min}$ after METH treatment, rats were placed into the open field and locomotor activity was measured for $3 \mathrm{~h}$. Four rats were tested concurrently in four separate open fields. Locomotor activity was estimated as distance traveled and recorded in $20 \mathrm{~min}$ bins.

For the sensitization experiments, six groups of rats $(n=5-6$ per group) were habituated to the open field during $30 \mathrm{~min}$ 
on two consecutive days. Because this experiment assessed the chronic effects of RO5203648 and METH, the doses selected were lower than that used in the 3-h locomotor experiment described above. During the 14 days sensitization period, rats received daily treatment of RO5203648 (0, 1.67, $5 \mathrm{mg} / \mathrm{kg}$, i.p.) followed $10 \mathrm{~min}$ after by METH $(0,0.75 \mathrm{mg} / \mathrm{kg}$, i.p.) and were allowed to freely explore the open field for $60 \mathrm{~min}$. Four rats were tested concurrently in four separate open fields. Locomotor activity was recorded on alternate days. Treatments were administered in the home cages on the no-test days. Rats underwent withdrawal from all pharmacological treatments for three consecutive days before receiving a challenge with a low dose of $\mathrm{METH}(0.25 \mathrm{mg} / \mathrm{kg}$, i.p. $)$ to probe for sensitization. Locomotor activity was recorded for $60 \mathrm{~min}$ in the sensitization test.

\section{S-A experiments}

Eight operant conditioning chambers (Panlab, SL, Barcelona, Spain) controlled by software (Packwin software package) were used in the S-A experiments. Chambers were equipped with two response levers, an infusion pump, a house light and a stimulus light. Presses on the active lever resulted in activation of the infusion pump and delivery of METH or saline, illumination of a light stimulus for $5 \mathrm{~s}$ and initiation of a $20 \mathrm{~s}$ time-out. Presses on the inactive lever were recorded but had no programmed consequences. Each experimental chamber was enclosed in a lightand sound-attenuating box. The house light was on throughout training and test sessions.

After surgery, rats were randomly assigned to METH $(n=10)$ and saline groups $(n=6)$ and were trained to receive METH $(0.05 \mathrm{mg} / \mathrm{kg} /$ infusion$)$ or saline infusions under a fixed ratio (FR) 1 reinforcement schedule in daily $60 \mathrm{~min}$ sessions. METH intake tests began after the rats in the METH group met a criterion of consistency and stability (number infusions per session $\geq 15$ for three consecutive days with less than $20 \%$ variability). Each rat completed three METH-taking tests on alternate days during which a pre-treatment of $\operatorname{RO} 5203648(0,3,10 \mathrm{mg} / \mathrm{kg}$, i.p.) was administered $10 \mathrm{~min}$ before the self-administration session. The order in which treatments were administered was counterbalanced between subjects. After completion of all three tests rats were re-exposed to standard METH or saline self-administration sessions.

After the rats in the METH group returned to stable responding (number infusions per session $\geq 15$ ), we tested selfadministration of the TAAR1 partial agonist to examine its reinforcing properties. Each rat underwent four substitution tests in which varying doses of RO5203648 were given through intravenous infusions $(0,0.25,0.5$, and $1 \mathrm{mg} / \mathrm{kg} /$ infusion $)$. Substitution tests were conducted on separate $60 \mathrm{~min}$ sessions, one for each dose, on alternate days. The order of the four tests was fully randomized. After all four substitution tests were carried out, rats completed a test in which the selfadministered solution was replaced by a dose of METH three times weaker than the training dose $(0.017 \mathrm{mg} / \mathrm{kg} /$ infusion; training dose was $0.05 \mathrm{mg} / \mathrm{kg} /$ infusion). This test was done in order to ascertain that the rats remained sensitive to variations in the reinforcing efficacy of the drug available for self-administration.
To test for potential nonspecific effects of RO5203648 on general motivation and performance rats were trained on saccharin $(0.1 \%)$ self-administration. After two sessions of stable responding ( $\geq 15$ reinforcements per session), each rat was tested three times, receiving a pretreatment of $\mathrm{RO} 5203648(0,3,10 \mathrm{mg} / \mathrm{kg}$ i.p.), administered in a counterbalanced fashion $10 \mathrm{~min}$ before the saccharin self-administration session started.

\section{SYNAPTOSOME [ $\left.{ }^{3} \mathrm{H}\right]$ DA UPTAKE AND EFFLUX ASSAYS}

Wistar rats were sacrificed at 12 weeks of age to collect brain samples. This strain was used because of the availability and reliability of standards on our extensive database and consistent results with a range of psychomotor stimulants. For each synaptosomal preparation we pooled 5 rat brains. Each experiment was performed in 3 technical replicates and 3-5 biological replicates. The striatum was dissected out and homogenized in 20 vol buffer A $(0.32 \mathrm{M}$ sucrose, $4 \mathrm{mM}$ Hepes/ $\mathrm{NaOH}, \mathrm{pH} 7.4$, $5 \mathrm{mM}$ DTT, $1 \mathrm{mM}$ EDTA and complete protease inhibitor) with a homogenizer using ceramic beads (Minilyser, Bertin technologies, France). The homogenate was centrifuged at $800 \times$ g at $4^{\circ} \mathrm{C}$ for $10 \mathrm{~min}$ and the supernatant was kept. The pellet was resuspended in 5 vol buffer A and centrifuged again under the same conditions. Supernatants were pooled and centrifuged at $9000 \times \mathrm{g}$ at $4^{\circ} \mathrm{C}$ for $20 \mathrm{~min}$. The pellet was resuspended in 10 vol buffer $\mathrm{A}$ and centrifuged again under the same conditions. The pellet was resuspended in $1 \mathrm{ml} /$ brain recovery cell culture freezing media (LuBioScience, Lucerne, Switzerland). Protein concentration was determined and stored at $-80^{\circ} \mathrm{C}$ until needed.

On the day of the uptake assay, striatal synaptosomes were washed in Krebs-Hepes buffer, incubated for $20 \mathrm{~min}$ at room temperature with various concentrations of METH (0.00003$30 \mu \mathrm{M})$ with or without co-application of $3 \mu \mathrm{M}$ RO5203648, or with various concentrations of RO5203648 alone (0.00003$30 \mu \mathrm{M})$. The synaptosomes were then incubated with DA tracer for $10 \mathrm{~min}$. After rigorous washing with PBS, scintillator (Perkin Elmer, Schwerzenbach, Switzerland) was added and the uptake of $\left[{ }^{3} \mathrm{H}\right]$ DA was detected with a liquid scintillation analyzer (TopLab, Switzerland).

For the efflux assay, synaptosomes were preloaded with $\left[{ }^{3} \mathrm{H}\right] \mathrm{DA}$ and incubated in Krebs-Hepes buffer at $25^{\circ} \mathrm{C}$ for $20 \mathrm{~min}$. After centrifuged at $4^{\circ} \mathrm{C}$ for $3 \mathrm{~min}$ (max speed), the synaptosomes were resuspended in buffer containing different concentrations of METH $(0.3-10 \mu \mathrm{M})$ with or without co-application of $3 \mu \mathrm{M}$ RO5203648. The synaptosomes were then incubated at $25^{\circ} \mathrm{C}$ for $45 \mathrm{~min}$, washed again with ice cold buffer, and centrifuged, as previously, before scintillator was added and counted.

\section{IN VIVO MICRODIALYSIS}

In vivo microdialysis was performed in the right nucleus accumbens (NAc) of freely moving rats, using concentric microdialysis probes (membrane length $2 \mathrm{~mm}$, cut-off $6000 \mathrm{Da}$; CMA-11, CMA/Microdialysis, Solna, Sweden). Animals were anesthetized with oxygen/isofluran mixture, and positioned in a stereotaxic apparatus. Stereotaxic coordinates for probes position were chosen according to the rat brain atlas: $\mathrm{AP}+1.7$; 1.4 ; DV -8.1 relative to bregma. Probes were implanted in the brain vertically through small drilled aperture in the skull and fixed with dental 
cement. The dialysis probes were perfused during implantation into the brain and for $1 \mathrm{~h}$ afterward with artificial cerebrospinal fluid (aCSF) ( $\mathrm{NaCl} 147 \mathrm{mM}, \mathrm{KCl} 2.7 \mathrm{mM}, \mathrm{CaCl}_{2} 1.2 \mathrm{mM}, \mathrm{MgCl}_{2}$ $0.85 \mathrm{mM}$; CMA Microdialysis).

Approximately $24 \mathrm{~h}$ after surgery the dialysis probes were connected to a syringe pump and perfused with aCSF at $1.0 \mu \mathrm{l} / \mathrm{min}$ for $60 \mathrm{~min}$ (equilibration period) and then the perfusate was collected at a perfusion rate of $1.0 \mu \mathrm{l} / \mathrm{min}$ every $20 \mathrm{~min}$ for at least $60 \mathrm{~min}$ into collection tubes containing $2 \mu \mathrm{l}$ of $1 \mathrm{M}$ perchloric acid (basal levels). After this period animals were injected with vehicle, METH (0.75 mg/kg, i.p.) or RO5203648 $(5 \mathrm{mg} / \mathrm{kg}$, i.p.) or received double injections with RO5203648 and METH together (at the same doses) and the perfusate was collected at a perfusion rate of $1.0 \mu \mathrm{l} / \mathrm{min}$ every $20 \mathrm{~min}$ over a $3 \mathrm{~h}$ period. Quantification of DA in microdialysis samples was performed using HPLC with electrochemical detection (ALEXYS LC-EC system) equipped with a reverse-phase column ( $3 \mu \mathrm{m}$ particles, ALB-215 C18, $1 \times 150$ mm, Antec Leyden BV, Netherlands) at a flow rate of $200 \mu \mathrm{l} / \mathrm{min}$ and electrochemically detected by a $0.7 \mathrm{~mm}$ glass carbon electrode (Antec; VT-03). The mobile phase contained $50 \mathrm{mM} \mathrm{H}_{3} \mathrm{PO}_{4}, 50 \mathrm{mM}$ citric acid, $8 \mathrm{mM} \mathrm{KCl}$, $0.1 \mathrm{mM}$ EDTA, $400 \mathrm{mg} / \mathrm{l}$ octanesulfonic acid sodium salt and 10\% ( vol/vol) methanol, $\mathrm{pH} 3.9$. The sensitivity of the method permitted detection of $\sim 3$ fmol DA. Dialysate samples $(11 \mu \mathrm{l})$ were injected into HPLC without any purification.

\section{STATISTICAL ANALYSIS}

Data were analyzed by analysis of variance (ANOVA) with repeated measures when a within-subjects design was in use. Posthoc comparisons were conducted with the method of NewmanKeuls $(\mathrm{N}-\mathrm{K})$ using the sampling error from the overall ANOVA as denominator. Statistical significance was set at $\alpha=0.05$ for all experiments. All statistical analyses were performed using StatView 5.0 (SAS Institute, NC, USA).

\section{RESULTS}

\section{R05203648 BLOCKS METH-INDUCED LOCOMOTOR SENSITIZATION}

Rats were chronically treated with RO5203648 (0, 1.67, $5 \mathrm{mg} / \mathrm{kg}$, i.p.), administered $15 \mathrm{~min}$ before $\operatorname{METH}(0,0.75 \mathrm{mg} / \mathrm{kg}$, i.p.), and locomotor activity was measured on alternate days. RO5203648 significantly attenuated locomotor sensitization induced by repeated METH treatment (Figure 1A). A repeated measure ANOVA yielded a significant main effect of drug treatment $\left(F_{5,28}=26.68, p<0.0001\right)$ and a significant interaction effect between the factors drug treatment, session ( 1 through to 7 ) and time (twelve 5 min time bins) $\left(F_{330,1848}=1.19, p<0.019\right)$. Post-hoc comparisons showed that METH significantly enhanced locomotor activity as treatments progressed, with activity of the 7 th test being significantly higher compared with the 1 st $(p<$ 0.01 , by $\mathrm{N}-\mathrm{K}$ tests). The ability of RO5203648 to attenuate the effects of METH gradually increased across sessions. Both doses of RO5203648 were effective at reducing METH-induced locomotor activity $(p<0.05$ for session 2 and $4, p<0.01$ for sessions 5 through to 7 ) and preventing METH sensitization during the acquisition phase (Figure 1A).

After 14 days sensitization treatment and 3-day withdrawal rats received METH $(0.25 \mathrm{mg} / \mathrm{kg}$, i.p. $)$ and locomotor activity was evaluated. Previous repeated METH exposure in the sensitization phase led to a significantly heightened response to METH administration, which was significantly attenuated by RO5203648 (Figure 1B). ANOVA revealed a significant effect of treatment $\left(F_{5,28}=3.27, p=0.0189\right)$. Post-hoc comparisons indicated that rats with a history of chronic METH treatment showed significantly higher locomotor activity comparing to controls $(p<$

$$
\begin{aligned}
& \text { A } \\
& -0 \text { RO }-1.67 \text { RO }-5 \text { RO } \\
& -\square \text { MA }-1.67 \text { ROMA }-5 \text { RO-MA }
\end{aligned}
$$
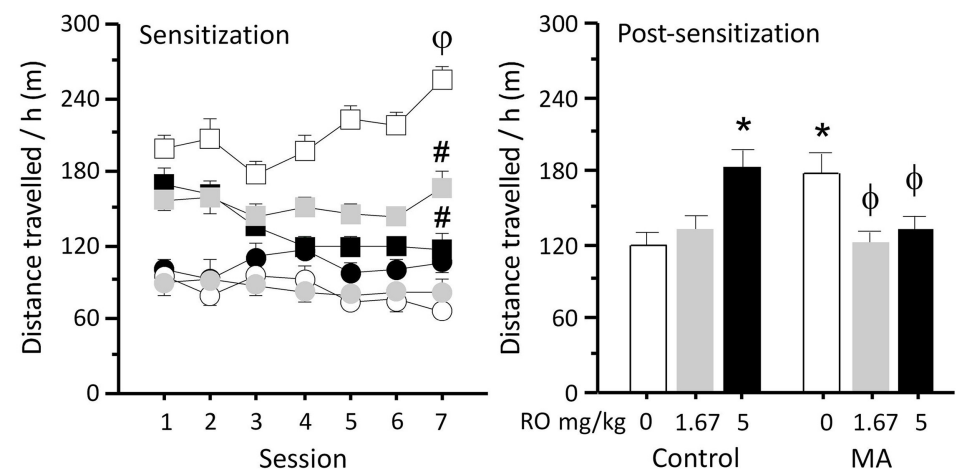
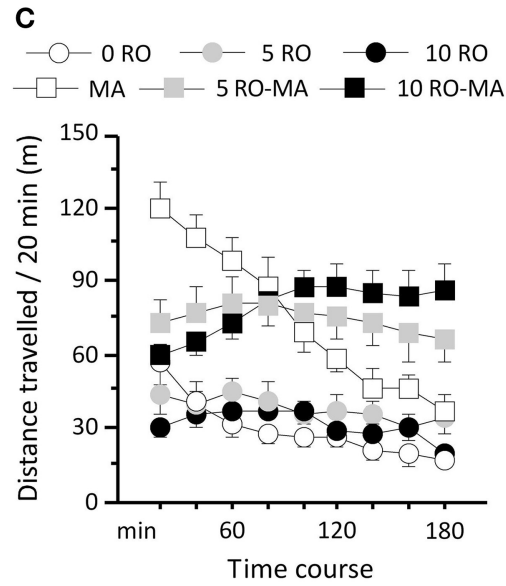

FIGURE 1 | Modulation of METH-induced locomotor activity and sensitization by TAAR1. (A) Chronic exposure to methamphetamine gradually increased locomotor activity. Low and moderate doses of RO5203648 attenuated METH-induced locomotor activity and blocked the progressive increment induced by repeated exposure to METH. (B) Following withdrawal from chronic METH exposure, a challenge with a low dose of METH produced sensitized locomotor responsiveness. The long-term expression of locomotor sensitization was prevented in rats that had received concurrent treatment with R05203648 (at either low or moderate doses) and METH. Treatment with the high dose of R05203648 alone during the sensitization phase enhanced the subsequent long-term response to METH. (C) METH produced robust increases in locomotor activity compared with control treatment in a 3-h activity test. R05203648 produced an early attenuation and a late potentiation of METH-stimulated locomotor activity. $\varphi p<0.01$, different from acute METH (day 1); ${ }^{\#} p<0.01$, different from chronic METH (day 7), * $p<0.01$ different from METH (after chronic control treatment), $\phi p<0.01$ different from METH (after chronic

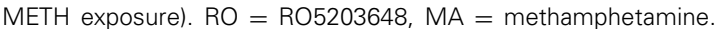


0.01 , by $\mathrm{N}-\mathrm{K}$ tests). This sensitized response was significantly reduced by both doses of RO5203648 ( $p<0.01$, by N-K tests). Moreover, RO5203648 treatment alone at the high dose, but not the low dose, produced significant cross-sensitization in the probe test as revealed by post-hoc comparisons $(p<0.01$ comparing to control, by $\mathrm{N}-\mathrm{K}$ tests).

\section{R05203648 TIME-DEPENDENTLY MODULATES METH-INDUCED LOCOMOTOR ACTIVITY}

To study the interactions between RO5203648 and METH across time, locomotor activity induced by these treatments was examined for $3 \mathrm{~h}$ in an open field following single and combined administration. Rats received a pre-treatment of RO5203648 $(0,5$ or $10 \mathrm{mg} / \mathrm{kg}$, i.p.) $15 \mathrm{~min}$ before METH (0 or $0.75 \mathrm{mg} / \mathrm{kg}$, i.p.). RO5203648 altered the effects of METH on locomotor activity in a time-dependent fashion, producing an early attenuation followed by a striking late potentiation. Locomotor activity was analyzed by repeated-measure ANOVA, which showed a significant effect of treatment $\left(F_{5,29}=15.80, p<0.0001\right)$ and time $\left(F_{8}, 232=15.94, p<0.0001\right)$, as well as a significant interaction between these factors $\left(F_{40,232}=8.35, p<0.0001\right)$ (Figure 1C). METH produced high levels of locomotor activity in the first $20 \mathrm{~min}$ that decreased gradually over the $3 \mathrm{~h}$ test. RO5203648 significantly attenuated METH-induced hyperlocomotion during the first hour at both the low and the high doses ( $p<0.01$ for the 1 st -3 rd bins, by N-K tests). However, as the effect of METH treatment alone slowly subsided, RO5203648 potentiated METH's effects by maintaining locomotor activity at a moderately high levels, which were significantly higher than that produced by METH alone at both the low ( $p<0.05$ for the 6 th bin, $p<0.01$ for the 7-9th bins, by N-K tests) and the high dose of RO5203648 ( $p<0.05$ for the 5th bin, $p<0.01$ for the 6-9th bins, by $\mathrm{N}-\mathrm{K}$ tests).

\section{R05203648 BLOCKS METH, BUT NOT SACCHARIN} SELF-ADMINISTRATION, AND LACKS REINFORCING EFFICACY

We then tested the ability of RO5203648 to attenuate METH S-A. Rats trained to self-administer METH under a FR1 schedule of reinforcement obtained a significantly higher number of infusions than rats responding for saline $(n=6)\left(F_{1,11}=34.45\right.$, $p=0.0001)$ (Figure 2A). Pre-treatment with $\operatorname{RO5203648}(0,3$, $10 \mathrm{mg} / \mathrm{kg}$, i.p.) significantly attenuated METH S-A (Figure 1B). ANOVA revealed a significant main effect of drug (METH vs. saline, $F_{1,10}=23.39, p=0.0007$ ) and dose of RO5203648 $\left(F_{2,20}=12.12, p=0.0004\right)$, as well as a significant interaction between those factors $\left(F_{2,20}=9.74, p=0.0011\right)$. Post-hoc comparisons indicated significant effects of both doses of RO5203648 on the number of METH infusions obtained ( $p<0.01$, by $\mathrm{N}-\mathrm{K}$ tests) (Figure 2B). The number of infusions obtained by the control group remained low and was not affected by RO5203648.

To control for potential motoric and motivational confounds, we tested the effects of RO5203648 on responding maintained by a natural reinforcer (saccharin). Pre-treatment with RO5203648 $(0,3,10 \mathrm{mg} / \mathrm{kg}$, i.p. $) 10 \mathrm{~min}$ before the saccharin S-A session did not affect responding as revealed by a repeated measures ANOVA $(p=0.639)$ (Figure 2C).

To test the abuse potential of RO5203648, we substituted METH for RO5203648 in the S-A task. Rats received infusions of RO5203648 at different doses $(0,0.25,0.5,1.0 \mathrm{mg} / \mathrm{kg} / \mathrm{infusion}$, i.v.) on separate tests. In the final test rats worked for METH infusions $(0.017 \mathrm{mg} / \mathrm{kg} /$ infusion $)$. One-way ANOVA revealed a significant effect of the self-administered drug $\left(F_{4,24}=11.67\right.$, $p<0.0001$ ) (Figure 2D). Post-hoc comparisons showed that S-A of different doses of RO5203648 did not generate varying levels of responding over and above S-A of saline. However, S-A of METH maintained significantly elevated rates of responding compared with RO5203648 and saline infusions ( $p<0.01$, by N-K tests), with the number of METH infusions at the low dose being 2-fold greater than that obtained with the training dose (Figure 2B).

\section{R05203648 HAS NO EFFECT ON METH-INDUCED DA RELEASE OR INHIBITION OF DA UPTAKE IN THE STRIATUM IN VITRO}

In order to investigate the impact of TAAR1 partial agonism on METH-induced DA release and uptake, striatal synaptosomes were stimulated with various concentrations of METH to
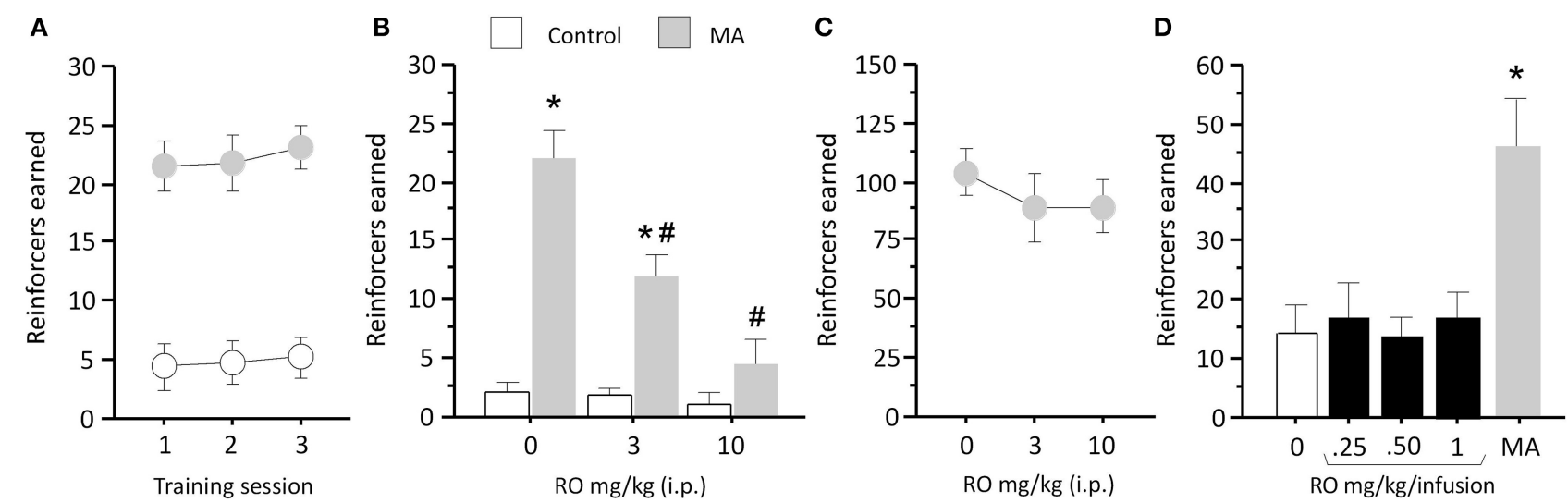

FIGURE 2 | R05203648 decreases METH S-A and exhibits reduced abuse liability in a substitution test. (A) Rats were trained on METH S-A until consistent performance was attained. (B) R05203648 dose-dependently reduced METH S-A but did not affect sucrose S-A (C). (D) In a substitution procedure, varying concentrations of

R05203648 did not sustain S-A behavior over and above control values, whereas a concentration of METH three times lower than that used during training generated strong responding. ${ }^{*} p<0.01$, different from control values; ${ }^{\#} p<0.01$, different from METH. RO $=$ R05203648, MA $=$ methamphetamine. 
elevate synaptic levels of DA. METH-induced increases in DA transmission were not affected by the co-application of $3 \mu \mathrm{M}$ RO5203648, as revealed by a two-way ANOVA which showed only a significant main effect of the METH treatment $\left(F_{3,14}=4.56\right.$, $p=0.0199$ ) (Figure 3A). In the uptake assay, METH induced a significant inhibition of DA uptake in a concentration-dependent manner. Similarly, uptake inhibition was not altered by the coincubation with $3 \mu \mathrm{M}$ RO5203648. RO5203648 alone also produced a weak DA uptake inhibition, but at a concentration $\left(\mathrm{IC}_{50}\right.$ $3 \mu \mathrm{M}) 10$-folder higher than that produced by METH alone $\left(\mathrm{IC}_{50}\right.$ $0.3 \mu \mathrm{M})$. A Two-Way ANOVA revealed a significant main effect of concentration $\left(F_{10,44}=89.37, p<0.0001\right)$ and a significant main effect of treatment $\left(F_{2,44}=34.23, p<0.0001\right)$, as well as a significant interaction between those factors $\left(F_{20,44}=2.70\right.$, $p=0.0030)$ (Figure 3B).

\section{R05203648 ATTENUATES METH-INDUCED CHANGES IN DA TRANSMISSION IN THE NAC}

To explore the mechanisms underlying the ability of RO5203648 to modulate METH-stimulated behaviors, we measured DA outflow in the NAc using in vivo microdialysis. Samples were collected for $3 \mathrm{~h}$ at $20 \mathrm{~min}$ intervals following treatment with METH, alone and in combination with RO5203648. A repeated measure ANOVA revealed a significant effect of treatment $\left(F_{3,23}=20.16\right.$, $p<0.0001)$ and time $\left(F_{9,207}=13.47, p<0.0001\right)$, as well as a significant interaction between these factors $\left(F_{27,207}=5.35\right.$, $p<0.0001)$. Post-hoc comparisons showed that METH produced a significant increase in DA level, which reached peak level at $60 \mathrm{~min}$, followed by a decrease over time $(p<0.01$ comparing to control, for the 1st-6th bins, by N-K tests). RO5203648 blocked the METH-induced increase in DA levels in the first $20 \mathrm{~min}$ after METH administration ( $p<0.01$ at $40 \mathrm{~min}$, by $\mathrm{N}-\mathrm{K}$ tests) (Figure 3C).

\section{DISCUSSION}

The present data provide extensive evidence of interactions between METH and the TAAR1 partial agonist, RO5203648, demonstrated in a range of behavioral paradigms and neurochemical experiments, including tests of locomotor activity, sensitization, self-administration, and in vivo microdialysis. Taking together, the current data showed a significant ability of TAAR1 to modulate the neurochemical and behavioral effects of METH.

METH is known for its ability to potently increase extracellular DA levels in the mesolimbic dopaminergic pathway, which largely underlies its motor-stimulating and rewarding effects. Following repeated exposure, METH produces long-lasting neuroadaptive changes in the mesolimbic DA system, characterized by a progressive augmentation of stimulant-induced DA efflux, which is thought to be a key neurochemical signature of METH behavioral sensitization (Yamada et al., 1988; Nakagawa et al., 2011). Our finding that repeated RO5203648 co-administered with METH blocked the development of METH sensitization suggests that TAAR1 is able to modulate the long-term neuroadaptations induced by chronic METH exposure. In line with these observations, it has been shown previously that mice lacking taar 1 were hypersensitive to the motor-stimulating effects of METH (Achat-Mendes et al., 2012), suggesting that TAAR1 is either constitutively active or tonically activated by ambient ligands to suppress the dopaminergic response to METH. In the current study, TAAR1 activation by RO5203648 may have exerted an inhibitory control over METH-evoked DA transmission, thereby preventing the induction of the dopaminergic hypersensitivity that underlies METH sensitization. Markedly complicating this picture, however, is the fact that METH itself is a potent full agonist at TAAR1, potentially leading to the initiation of phosphorylation events that down-regulate DAT function resulting in extracellular DA accumulation (Xie and Miller, 2009). Hence, alternatively or complementarily, RO5203648 may block METH-induced excessive DA transmission through the TAAR1mediated pathway by directly competing with METH at TAAR1, thereby attenuating the progression of dopaminergic sensitization induced by repeated METH. Intriguingly, repeated RO5203648 alone, at moderate doses $(5 \mathrm{mg} / \mathrm{kg})$, cross-sensitized with $\mathrm{METH}$,
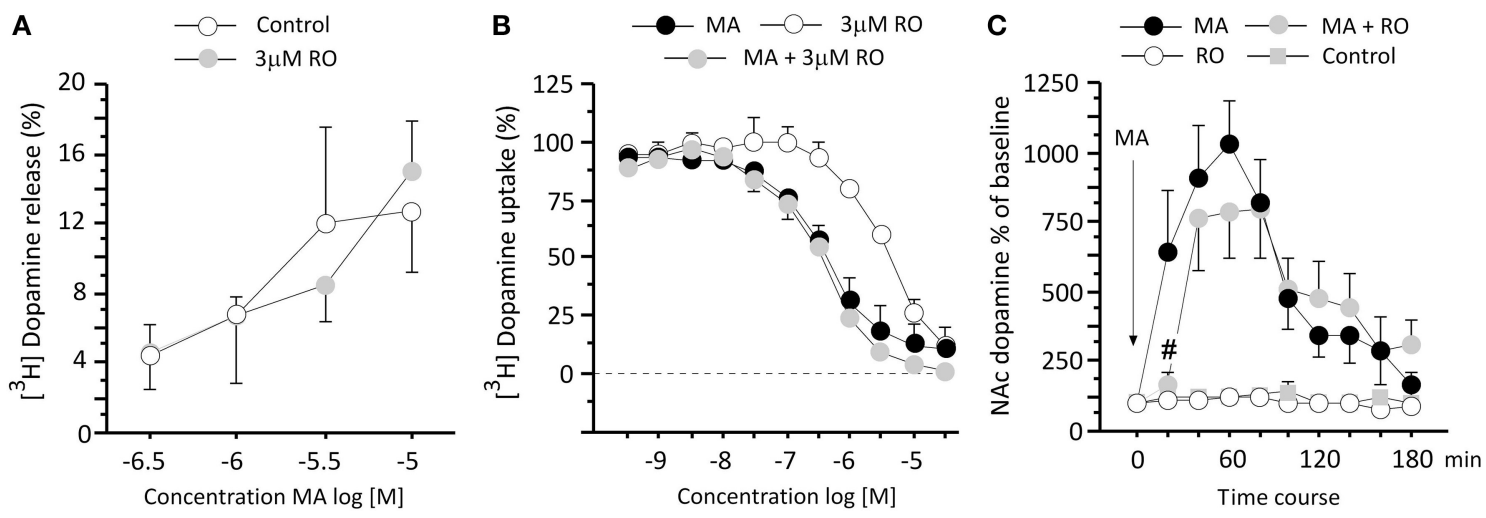

FIGURE 3 | RO5203648 transiently attenuated DA overflow in the NAc in vivo but not in striatal synaptosomes. (A) In striatal synaptosomes the efflux of $\left[{ }^{3} \mathrm{H}\right] \mathrm{DA}$ induced by varying concentrations of METH was not affected by co-application of RO5203648, as was the uptake of $\left[{ }^{3} \mathrm{H}\right] \mathrm{DA}$ (B) In the 3-h long in vivo microdialysis experiment R05203648 delayed the strong increases in extracellular DA accumulation induced by METH treatment in the NAc, producing a marked transient inhibition of the DA response $(\mathbf{C})$ please note that a pure control cannot be included in the uptake experiments $(\mathbf{B})$ because no efflux or release can be measured after vehicle treatment. ${ }^{\#} p<0.01$, different from METH. $\mathrm{RO}=\mathrm{RO} 5203648, \mathrm{MA}=$ methamphetamine 
suggesting that long-term treatment with the partial agonist may have resulted in neuroadaptations in the mesolimbic DA system that are similar to those evoked by chronic METH treatment. Previous studies have shown that alterations of both D1 and D2 DA receptors play a key role in the development of stimulant sensitization (Shuto et al., 2006; McGinty et al., 2008). Although there is no evidence of direct interactions of TAAR1 with DA D1 receptors, previous data hints at a complex inter-relationship between TAAR1 and DA D2 receptors. Xie et al. (2008) demonstrated cross-attenuation of signaling between TAAR1 and DA D2 receptors, with their signaling having opposite effects on intracellular phosphorylation cascades and extracellular DA transmission (Xie et al., 2008). Additional findings revealed that taar1 knockout mice displayed up-regulation of high-affinity striatal D2 receptors (Wolinsky et al., 2007) and reduced D2 receptormediated auto-inhibition (Leo et al., 2014). Thus, it is possible that intermittent partial activation of TAAR1 with RO5203648 alters the coordinated interactions between TAAR1 and DA D2 receptors or causes neuroadaptive changes in DA D2 receptors, leading to altered auto-inhibitory control over dopaminergic transmission. Alternatively or complementarily, repeated TAAR1 activation may lead to persistent changes in glutamatergic transmission. Enhanced glutamatergic transmission in the ventral tegmental area and nucleus accumbens plays a fundamental role in stimulant sensitization (Wolf, 1998; Vanderschuren and Kalivas, 2000). The full TAAR1 agonist, RO5166017, prevented the hyperactivity induced by NMDA receptor blockade (Revel et al., 2011), suggesting that TAAR1 activation may facilitate glutamate neurotransmission. Importantly, at the low dose $(1.67 \mathrm{mg} / \mathrm{kg}$ ) RO5203648 did not cross-sensitize with METH but maintained its ability to reduce METH sensitization. Therefore, it would appear that at low doses the partial TAAR1 agonist is able to block METH sensitization without inducing long-term METH-like neuroadaptations.

To further investigate the interactions between TAAR1 and $\mathrm{METH}$, we conducted a 3-h locomotor activity test after acute treatment with RO5203648, alone and combined with METH. Early during the test RO5203648 produced a dose-dependent attenuation of METH-induced hyperactivity, which is consistent with our finding that partial TAAR1 activation attenuated METH-evoked DA release. Interestingly, RO5203648 potentiated the locomotor-enhancing effects of METH in the later phase of the locomotor activity test, as METH's effects began to decay. Our attempt to gain insight into the neurochemical mechanisms mediating this interaction was only partially successful. METHinduced DA accumulation in the NAc seemed to be altered by TAAR1 activation in biphasic fashion but the late potentiation effect on DA overflow did not reach statistical significance. This indicates that mechanisms other than net DA transmission at NAc synapses are likely to contribute to the complex regulation of METH-induced hyperactivity by TAAR1. Additionally, the later increase in locomotion is unlikely to be explained by a gradual temporal decay of RO5203648's effects, which could potentially allow the recovery of METH's effects over time, since this biphasic action of RO5203648 was also observed in a previous study in which RO5203648 alone produced an early inhibition followed by a later enhancement of food-maintained responding
(Pei et al., 2014). In the experiment by Pei et al. rats treated with RO5203648 only (i.e., not combined with METH) responded for food over and above control rats under a progressive ratio schedule of reinforcement, with more responses accumulating long after treatment (3-6h after), suggesting that RO5203648 remains psychoactive for several hours.

In the synaptosomal preparation RO5203648 did not affect METH-induced striatal DA release and DA uptake inhibition, suggesting that RO5203648 regulation of METH's behavioral effects is unlikely to depend on direct, local actions at the DAT. As previously indicated, the in vivo microdialysis data revealed transient but significant reduction in METH-induced DA overflow following RO5203648 treatment. The apparent discrepancy between the in vitro and in vivo experiments could be explained by the difference in concentrations used and the network effects of systemic TAAR1 activation. Indeed, TAAR1 seems to exert inhibitory control over the activity of DA-releasing neurons originating in the ventral tegmental area. The full TAAR1 agonist, RO5166017, decreased the firing rate of mouse midbrain DA neurons (Revel et al., 2011), whereas the effects of RO5203648 on DA transmission appeared to be dependent on ambient levels of endogenous agonists, including DA itself, given its partial agonist profile (Revel et al., 2012b). The synaptosomal assays were conducted with tissue that may have contained synaptic fractions from dorsal striatum, thus potentially adding variability to the assay. We also acknowledge the use of different strains of rat for the synaptosomal assays and microdialysis experiments. Thus, tissue-specific and strain-specific differences may have contributed to these inconsistent results.

The current findings also demonstrated that RO5203648 dose-dependently attenuated METH self-administration, which is likely to reflect a decrease in the reinforcing efficacy of METH, although we acknowledge that we only tested METH S-A under a FR1 schedule and at one dose $(0.05 \mathrm{mg} / \mathrm{kg} /$ infusion $)$. Our data are consistent with previous evidence indicating that the partial agonist RO5263397 reduced self-administration of METH at doses of METH that were both on the ascending and descending limbs of the dose-response curve (Jing et al., 2014). Further, we showed that the effect of RO5203648 on METH self-administration could not be attributed to general motivational or motor deficits because RO5203648 did not affect sucrose self-administration. Previous studies have shown that RO5203648 decreased cocaine self-administration and cocaine seeking at doses that did not impair food-maintained responding (Revel et al., 2012b; Pei et al., 2014). These observations are consistent with the finding that taar1 deletion led to earlier acquisition and delayed extinction of place preference induced by METH exposure (Achat-Mendes et al., 2012), similarly suggesting an inhibitory control of TAAR1 over METH's rewarding properties. Importantly, our data also revealed that RO5203648 did not maintain significant levels of $\mathrm{S}$-A when it was substituted for METH, which may be indicative of low abuse potential, a desirable feature for TAAR1-based pharmacotherapies to have. On the basis of these data, and considering that TAAR1 agonists have been shown to have clear therapeuticlike effects in models of cocaine addiction (Pei et al., 2014; Thorn et al., 2014), it may be tempting to speculate that such potential clinical application could be expanded to METH addiction. 
However, such generalization requires considerable caution given the complexities revealed by the present results, especially those that relate to long-term TAAR1 agonist treatment and behavioral sensitization.

In summary, the present data revealed novel complex interactions between the selective TAAR1 partial agonist, RO5203648, and METH. These findings further demonstrate the potential of TAAR1 to modulate stimulant-induced neurochemical and behavioral effects and provide additional support for the investigation of TAAR1 as a target for therapeutic intervention in addictive disorders.

\section{ACKNOWLEDGMENTS}

This work was supported by grants to J. J. C. from the Spanish Ministry of Health (ISCIII, grant PI10/00297) and F. Hoffman-La Roche Ltd (Grant Number RM43G0283), and to R. R. G. from the Russian Science Foundation (project N14-25-00065). We thank Ana M. Muñoz for technical assistance.

\section{REFERENCES}

Achat-Mendes, C., Lynch, L. J., Sullivan, K. A., Vallender, E. J., and Miller, G. M. (2012). Augmentation of methamphetamine-induced behaviors in transgenic mice lacking the trace amine-associated receptor 1. Pharmacol. Biochem. Behav. 101, 201-207. doi: 10.1016/j.pbb.2011.10.025

Berry, M. D. (2004). Mammalian central nervous ssystem trace amines. Pharmacologic amphetamines, physiologic neuromodulators. J. Neurochem. 90, 257-271. doi: 10.1111/j.1471-4159.2004.02501.x

Borowsky, B., Adham, N., Jones, K. A., Raddatz, R., Artymyshyn, R., Ogozalek, K. L., et al. (2001). Trace amines: identification of a family of mammalian G protein-coupled receptors. Proc. Natl. Acad. Sci. U.S.A. 98, 8966-8971. doi: 10.1073/pnas. 151105198

Bradaia, A., Trube, G., Stalder, H., Norcross, R. D., Ozmen, L., Wettstein, J. G., et al. (2009). The selective antagonist EPPTB reveals TAAR1-mediated regulatory mechanisms in dopaminergic neurons of the mesolimbic system. Proc. Natl. Acad. Sci. U.S.A. 106, 20081-20086. doi: 10.1073/pnas.0906522106

Bunzow, J. R., Sonders, M. S., Arttamangkul, S., Harrison, L. M., Zhang, G., Quigley, D. I., et al. (2001). Amphetamine, 3,4methylenedioxymethamphetamine, lysergic acid diethylamide, and metabolites of the catecholamine neurotransmitters are agonists of a rat trace amine receptor. Mol. Pharmacol. 60, 1181-1188. doi: 10.1124/mol.60.6.1181

Elliott, J. M., and Beveridge, T. J. (2005). Psychostimulants and monoamine transporters: upsetting the balance. Curr. Opin. Pharmacol. 5, 94-100. doi: 10.1016/j.coph.2004.09.005

Espinoza, S., Salahpour, A., Masri, B., Sotnikova, T. D., Messa, M., Barak, L. S., et al. (2011). Functional interaction between trace amine-associated receptor 1 and dopamine D2 receptor. Mol. Pharmacol. 80, 416-425. doi: 10.1124/mol.111.073304

Fleckenstein, A. E., Volz, T. J., and Hanson, G. R. (2009). Psychostimulantinduced alterations in vesicular monoamine transporter-2 function: neurotoxic and therapeutic implications. Neuropharmacology 56(Suppl. 1), 133-138. doi: 10.1016/j.neuropharm.2008.07.002

Fleckenstein, A. E., Volz, T. J., Riddle, E. L., Gibb, J. W., and Hanson, G. R. (2007). New insights into the mechanism of action of amphetamines. Annu. Rev. Pharmacol. Toxicol. 47, 681-698. doi: 10.1146/annurev.pharmtox.47.120505.105140

Gilbert, D., and Cooper, S. J. (1983). beta-Phenylethylamine-, d-amphetamineand l-amphetamine-induced place preference conditioning in rats. Eur. J. Pharmacol. 95, 311-314. doi: 10.1016/0014-2999(83)90653-2

Goodwin, J. S., Larson, G. A., Swant, J., Sen, N., Javitch, J. A., Zahniser, N. R., et al. (2009). Amphetamine and methamphetamine differentially affect dopamine transporters in vitro and in vivo. J. Biol. Chem. 284, 2978-2989. doi: 10.1074/jbc.M805298200

Jing, L., Zhang, Y., and Li, J. X. (2014). Effects of the trace amine associated receptor 1 agonist RO5263397 on abuse-related behavioral indices of methamphetamine in rats. Int. J. Neuropsychopharm. doi: 10.1093/ijnp/pyu060. [Epub ahead of print].

Leo, D., Mus, L., Espinoza, S., Hoener, M. C., Sotnikova, T. D., and Gainetdinov, R. R. (2014). Taar1-mediated modulation of presynaptic dopaminergic neurotransmission: role of D2 dopamine autoreceptors. Neuropharmacology 81, 283-291. doi: 10.1016/j.neuropharm.2014.02.007

Lindemann, L., Meyer, C. A., Jeanneau, K., Bradaia, A., Ozmen, L., Bluethmann, H., et al. (2008). Trace amine-associated receptor 1 modulates dopaminergic activity. J. Pharmacol. Exp. Ther. 324, 948-956. doi: 10.1124/jpet.107. 132647

McGinty, J. F., Shi, X. D., Schwendt, M., Saylor, A., and Toda, S. (2008). Regulation of psychostimulant-induced signaling and gene expression in the striatum. J. Neurochem. 104, 1440-1449. doi: 10.1111/j.1471-4159.2008.05240.x

Nakagawa, T., Suzuki, Y., Nagayasu, K., Kitaichi, M., Shirakawa, H., and Kaneko, S. (2011). Repeated exposure to methamphetamine, cocaine or morphine induces augmentation of dopamine release in rat mesocorticolimbic slice co-cultures. PLoS ONE 6:e24865. doi: 10.1371/journal.pone.0024865

Pei, Y., Lee, J., Leo, D., Gainetdinov, R. R., Hoener, M. C., and Canales, J. J. (2014). Activation of the trace amine-associated receptor 1 prevents relapse to cocaine seeking. Neuropsychopharmacology 39, 2299-2308. doi: 10.1038/npp.2014.88

Reese, E. A., Bunzow, J. R., Arttamangkul, S., Sonders, M. S., and Grandy, D. K. (2007). Trace amine-associated receptor 1 displays species-dependent stereoselectivity for isomers of methamphetamine, amphetamine, and para-hydroxyamphetamine. J. Pharmacol. Exp. Ther. 321, 178-186. doi: 10.1124/jpet.106.115402

Revel, F. G., Meyer, C. A., Bradaia, A., Jeanneau, K., Calcagno, E., Andre, C. B., et al. (2012a). Brain-specific overexpression of trace amine-associated receptor 1 alters monoaminergic neurotransmission and decreases sensitivity to amphetamine. Neuropsychopharmacology 37, 2580-2592. doi: 10.1038/npp.2012.109

Revel, F. G., Moreau, J. L., Gainetdinov, R. R., Bradaia, A., Sotnikova, T. D., Mory, R., et al. (2011). TAAR1 activation modulates monoaminergic neurotransmission, preventing hyperdopaminergic and hypoglutamatergic activity. Proc. Natl. Acad. Sci. U.S.A. 108, 8485-8490. doi: 10.1073/pnas.1103029108

Revel, F. G., Moreau, J. L., Gainetdinov, R. R., Ferragud, A., Velazquez-Sanchez, C., Sotnikova, T. D., et al. (2012b). Trace amine-associated receptor 1 partial agonism reveals novel paradigm for neuropsychiatric therapeutics. Biol. Psychiatry 72, 934-942. doi: 10.1016/j.biopsych.2012.05.014

Shannon, H. E., and Thompson, W. A. (1984). Behavior maintained under fixed-interval and second-order schedules by intravenous injections of endogenous noncatecholic phenylethylamines in dogs. J. Pharmacol. Exp. Ther. 228, 691-695.

Shuto, T., Kuroiwa, M., Hamamura, M., Yabuuchi, K., Shimazoe, T., Watanabe, S., et al. (2006). Reversal of methamphetamine-induced behavioral sensitization by repeated administration of a dopamine D1 receptor agonist. Neuropharmacology 50, 991-997. doi: 10.1016/j.neuropharm.2006. 01.009

Sulzer, D., Sonders, M. S., Poulsen, N. W., and Galli, A. (2005). Mechanisms of neurotransmitter release by amphetamines: a review. Prog. Neurobiol. 75, 406-433. doi: 10.1016/j.pneurobio.2005.04.003

Thorn, D. A., Jing, L., Qiu, Y., Gancarz-Kausch, A. M., Galuska, C. M. Dietz, D. M., et al. (2014). Effects of the Trace Amine-Associated Receptor 1 Agonist RO5263397 on Abuse-Related Effects of Cocaine in Rats. Neuropsychopharmacology 39, 2309-2316. doi: 10.1038/npp.2014.91

Vanderschuren, L. J., and Kalivas, P. W. (2000). Alterations in dopaminergic and glutamatergic transmission in the induction and expression of behavioral sensitization: a critical review of preclinical studies. Psychopharmacology (Berl). 151, 99-120. doi: 10.1007/s002130000493

Velazquez-Sanchez, C., Ferragud, A., Ramos-Miguel, A., Garcia-Sevilla, J. A., and Canales, J. J. (2013). Substituting a long-acting dopamine uptake inhibitor for cocaine prevents relapse to cocaine seeking. Addict. Biol. 18, 633-643. doi: 10.1111/j.1369-1600.2012.00458.x

Velazquez-Sanchez, C., Ferragud, A., Renau-Piqueras, J., and Canales, J. J. (2011). Therapeutic-like properties of a dopamine uptake inhibitor in animal models of amphetamine addiction. Int. J. Neuropsychopharmacol. 14, 655-665. doi: $10.1017 /$ S1461145710000969

Wolf, M. E. (1998). The role of excitatory amino acids in behavioral sensitization to psychomotor stimulants. Prog. Neurobiol. 54, 679-720. doi: 10.1016/S03010082(97)00090-7 
Wolinsky, T. D., Swanson, C. J., Smith, K. E., Zhong, H., Borowsky, B., Seeman, P., et al. (2007). The TRACE Amine 1 receptor knockout mouse: an animal model with relevance to schizophrenia. Genes Brain Behav. 6, 628-639. doi: 10.1111/j.1601-183X.2006.00292.x

Xie, Z., and Miller, G. M. (2007). Trace amine-associated receptor 1 is a modulator of the dopamine transporter. J. Pharmacol. Exp. Ther. 321, 128-136. doi: 10.1124/jpet.106.117382

Xie, Z., and Miller, G. M. (2009). A receptor mechanism for methamphetamine action in dopamine transporter regulation in brain. J. Pharmacol. Exp. Ther. 330, 316-325. doi: 10.1124/jpet.109.153775

Xie, Z., Westmoreland, S. V., Bahn, M. E., Chen, G. L., Yang, H., Vallender, E. J., et al. (2007). Rhesus monkey trace amine-associated receptor 1 signaling: enhancement by monoamine transporters and attenuation by the D2 autoreceptor in vitro. J. Pharmacol. Exp. Ther. 321, 116-127. doi: 10.1124/jpet.106. 116863

Xie, Z., Westmoreland, S. V., and Miller, G. M. (2008). Modulation of monoamine transporters by common biogenic amines via trace amine-associated receptor 1 and monoamine autoreceptors in human embryonic kidney 293 cells and brain synaptosomes. J. Pharmacol. Exp. Ther. 325, 629-640. doi: 10.1124/jpet.107.135079

Yamada, S., Kojima, H., Yokoo, H., Tsutsumi, T., Takamuki, K., Anraku, S., et al. (1988). Enhancement of dopamine release from striatal slices of rats that were subchronically treated with methamphetamine. Biol. Psychiatry 24, 399-408. doi: 10.1016/0006-3223(88)90176-X

Conflict of Interest Statement: The authors declare that the research was conducted in the absence of any commercial or financial relationships that could be construed as a potential conflict of interest.

Received: 17 October 2014; accepted: 27 January 2015; published online: 13 February 2015.

Citation: Cotter R, Pei Y, Mus L, Harmeier A, Gainetdinov RR, Hoener MC and Canales JJ (2015) The trace amine-associated receptor 1 modulates methamphetamine's neurochemical and behavioral effects. Front. Neurosci. 9:39. doi: 10.3389/ fnins.2015.00039

This article was submitted to Neuropharmacology, a section of the journal Frontiers in Neuroscience.

Copyright (c) 2015 Cotter, Pei, Mus, Harmeier, Gainetdinov, Hoener and Canales. This is an open-access article distributed under the terms of the Creative Commons Attribution License (CC BY). The use, distribution or reproduction in other forums is permitted, provided the original author(s) or licensor are credited and that the original publication in this journal is cited, in accordance with accepted academic practice. No use, distribution or reproduction is permitted which does not comply with these terms. 